\title{
SPASTIC PARAPARESIS AS THE ONSET MANIFESTATION OF SPINOCEREBELLAR ATAXIA TYPE 7
}

\author{
Salomão da Cunha Linhares", Wagner Goes Horta ${ }^{3}$, Francisco Marcos Bezerra da Cunha ${ }^{3}$, \\ José Daniel Vieira Castro ${ }^{3}$, Antonio Carlos dos Santos ${ }^{2}$, Wilson Marques Jr ${ }^{7}$
}

Spinocerebellar ataxia type 7 (SCA7) is an autosomal dominant neurodegenerative disorder caused by an unstable CAG repeat in the coding region of ataxin-7 gene ${ }^{1}$. A wide variety of neurological manifestations have been described in these patients, including ataxic gait, visual loss, dysartria, nystagmus, ophthalmoplegia, dysphagia, dystonia, and pyramidal and extrapyramidal signs ${ }^{2}$.

Different combinations of these manifestations may be found in individual patients, either among as within families ${ }^{3}$, but the commonly described onset clinical manifestations are cerebellar ataxia and visual impairment ${ }^{4,5}$.

We report a molecular proven SCA7 patient whose initial manifestation was spastic paraparesis in the absence of any definite cerebellar or visual impairment at the initial phases of the disease.

\section{METHOD}

We have retrospectively analyzed the onset manifestations of all SCA7 patients whose molecular diagnosis was confirmed at the DNA Laboratory of The Department of Neurology of the School of Medicine of Ribeirão Preto, University of São Paulo. The affected members and their families were reevaluated by one of us (SL). This study was approved by the Research Ethics Committee of our hospital and all participants gave an written informed consent. The SCA7 expansion was tested as described in Linhares ${ }^{6}$.

\section{RESULTS}

We have found 44 SCA7 patients, from 3 different families. The most frequent onset manifestation was cerebellar ataxia, present in 23 patients, followed by visual loss, the initial complaint of other 20 patients. The last affected individual presented an unusual manifestation: spastic paraparesis. This patient, individual VI.12 on the presented family tree (Figure), was a 50-year-old man when first seen at our institution. He complained that his legs seemed to be stiff since his twenties but at the age of 43 years this stiffness progressively increased, impairing his walking abilities. He denied any other neurological manifestation, including sphincter or sexual dysfunction, visual abnormalities or loss of balance. On neurological examination, at age of 50 , sensation was normal, but his gait was clearly spastic and tonus was significantly increased in the lower limbs, where a mild pyramidal weakness was also detected. No atrophy or abnormal movement was observed. His tendon jerks were hyperreflexic in the lower limbs, increased in the upper limbs, and the plantar responses were extensor bilaterally. His jaw reflex was also

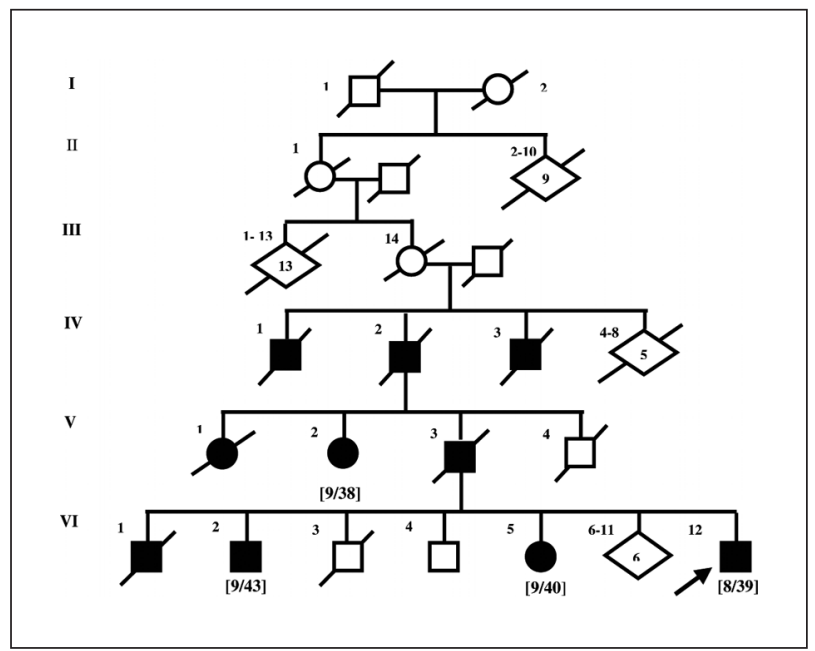

Figure. Brazilian family with spinocerebellar ataxia type $7: \bigcirc=$ unffected female; $\square=$ unffected male; $\mathbf{0}=$ affected female; $\mathbf{\square}=$ affected male; $\diamond=$ unffected male or female. The numbers inside the brackets indicate the size of the CAG repeats in each allele. The diagonal line across a symbol indicates a deceased individual. The arrow indicates the proband.

\section{PARAPARESIA ESPÁSTICA COMO MANIFESTAÇÃO INICIAL DA ATAXIA ESPINOCEREBELAR DO TIPO 7}

'Department of Neurology and 'Department of Internal Medicine, Division of Neuroradiology, School of Medicine at Ribeirão Preto, University of São Paulo, Ribeirão Preto SP, Brazil; ' 3 Department of Neurology, School of Medicine, Federal University of Ceara, Fortaleza CE, Brazil. This study was supported by grants from CNPq (Conselho Nacional de Pesquisa) and FAEPA (Fundação de Apoio ao Ensino, Pesquisa e Assistência do Hospital das Clínicas da Faculdade de Medicina de Ribeirão Preto).

Received 28 November 2007, received in final form 21 January 2008. Accepted 22 February 2008.

Dr. Wilson Marques Jr - Departamento de Neurologia / Faculdade de Medicina de Ribeirão Preto / Campus Universitário USP - 14048-900 Ribeirão Preto SP - Brasil. E-mail: wmjunior@fmrp.usp.br 
mildly increased. Contrasting to the exuberance of the pyramidal signs, on the finger-nose test there was a very mild decomposition and his ocular movements were also very mildly hypermetric, although saccades were preserved. No other abnormality was detected, including vision, hearing, pupilar reflexes, speech, memory and intellectual functions. At this time he was clinically diagnosed as having a spastic paraparesis syndrome, most probably a pure HSP. On evaluation of his family, however, it was noted that one of his aunts, individual $\mathrm{V}-2,72$ years old, was healthy until the age of 42 years when she first noticed a progressive visual loss. On examination, she was wheelchair bound, her speech was slurred and there was an evident limb dysmetria. The proband's brother (VI-2), 58 years, reported an 18-years history of unsteady gait and 10 years of bilateral progressive visual failure. At the examination, he was also wheelchair bound, presented a slurred speech and an exacerbated limb dysmetria. His sister (VI-5), 53 years, was also committed by loss of balance since she was 43 years old, and on examination we noted gait ataxia, limb dysmetria, dysartria and a moderate visual loss. Six affected individuals, including the probands father died before our evaluation, but it seems that in all of them the disease started with an ataxic gait. Laboratory investigation of the proband was normal to HTLV I and II, VDRL, FTA-abs (in serum and CSF), autoantibodies and CSF examination. A spinal cord MRI revealed only a mild disc protrusion at $\mathrm{C} 4-\mathrm{C} 5$ and $\mathrm{C} 5-\mathrm{C} 6$ levels, without spinal cord compression. A neurophysiologic screening for peripheral neuropathy was also normal. Based mainly on his family evaluation than in his own clinical manifestations, he was also submitted to a brain MRI that revealed a moderate atrophy of the cerebellar hemispheres. After informed consent, he and his available affected family members were tested to the SCA7 CAG expansion, due to the association of spinocerebellar manifestations and visual loss that turned out to be positive in all of them. The expansion sizes are depicted in brackets in Figure.

\section{DISCUSSION}

Involvement of the corticospinal tract is a common feature among of the autosomal dominant cerebellar ataxias, although a spastic gait has rarely been reported as an isolated onset manifestation. Sakai and Kawakami ${ }^{7}$ first described two siblings presenting spastic paraparesis in the early years of their disease that later developed the characteristic manifestations of the spinocerebellar ataxias. Since then other patients with similar clinical features have been described, respectively, by Kaneko ${ }^{8}$ and Teive ${ }^{9}$. All these patients were diagnosed as having SCA3/MJD disease. Previously, Sequeiros and Coutinho ${ }^{10}$ had observed that many patients at risk for Machado-Jo- seph disease (MJD) presented hyperreflexia on neurological examination, and that the first manifestation of the patients they studied resulted from pyramidal as well as cerebellar dysfunctions. Based on his own observations and those of Sequeiros and Coutinho, Sakai and Kawakami proposed that spastic paraplegia might be an SCA3/MJD under recognized onset manifestation. To the best of our knowledge no patient with SCA7 presenting isolated spastic paraparesis as the initial manifestation has been described so far and hyperreflexia does not seem to be a recognized abnormality in at risk individuals with this type of spinocerebellar degeneration ${ }^{2,11}$.

The normal range of the CAG repeats described in SCA7 disease ranges from 7 to 35 , and the lowest pathological number of repeats was established to be $37^{5}$. Johansson" verified that those patients presenting with visual impairment have a higher number of CAG repeats $(>59)$ while those beginning with ataxia have a lower number of repeats $(<59)$. In the Brazilian family we are reporting, all affected members had a low number of CAG repeats, ranging from 38 to 43 , although their onset manifestation varied among cerebellar ataxia, visual loss and spastic paraparesis. The observations made in this family show significant intra-familial phenotypic variability but do not reflect the influence of the number of the trinucleotide repeats in the nature of the initial symptom, as has been previously stated.

Instead of being diagnosed as having a spinocerebellar ataxia syndrome at the initial stages of his disease, the proband we describe was diagnosed as having an hereditary spastic paraparesis, a group of inherited disorders in which the main features are progressive gait disturbance, spasticity of the lower limbs, hiperreflexia, extensor plantar responses and a disproportionate mild pyramidal weakness ${ }^{12}$. Harding ${ }^{13}$ classified the HSP in two large groups, nominated as pure and complicated HSPs. In "pure" HSP the clinical manifestation are entirely dominated by the upper motor neuron syndrome, although minor abnormalities in cerebellar or sensory functions are admitted; while in "complicated" HSP many other systems may be involved in addition to spasticity, including optic atrophy, retinopathy, extrapyramidal disease, amyotrophy, dementia, ataxia, mental retardation, deafness, icthyosis, peripheral neuropathy and epilepsy. As is the case to SCA $3^{9}$, most patients with SCA7 must be differentiated from "complicated" HSP in the course of their disease, as both diseases show common manifestations, including ataxia, spasticity, retinopathy and extrapyramidal signs ${ }^{13}$. Occasionally, this distinction may be dificult in the course of the disease due to the absence of a clear predominance of the spasticity or of the cerebellar manifestations. The case we present, however, implies in a new clinical situ- 
ation. As has already been recognized to $\mathrm{SCA} 3^{7,8}, \mathrm{SCA} 7$ should also now be considered in the differential diagnosis of patients presenting with "pure" HSP. As this phenotype does not seem to run in all affected family members, evaluation of other affected sibs may direct to the diagnosis of SCA7 if there are members with visual impairment, or to both SCA7 and SCA3 if the affected members present a classic spinocerebellar syndrome.

In conclusion, it has already been recognized that clinical overlap between "complicated" forms of HSP and SCA3 and SCA7 should always be considered in the diagnosis process, but to this already known possibility we add the fact that SCA7 may also present syndromically as a "pure" HSP, and unless there are other diagnostic clues, mostly information from other affected members of the family, we think that all patients with the phenotype of spastic paraparesis should also be submitted to the DNA test for SCA7. This observation broadens the differential diagnosis of HSP, as also the neurological manifestations that can herald SCA7 disease, in addition to the classically recognized cerebellar ataxia and visual loss manifestations.

ACKNOWLEDGEMENTS - The authors thank Mrs. Sandra Elisabete Marques Nemoto for skillful laboratory work.

\section{REFERENCES}

1. David G, Abbas N, Stevanin G, et al. Cloning of the SCA7 gene reveals a highly unstable CAG repeat expansion. Nat Genet 1997;17:65-70.

2. David G, Giunti P, Abbas N, et al. The gene for autosomal dominant ataxia type II is located in a 5-cM in 3p12-13: genetic and physical mapping of the SCA7 locus. Am J Hum Genet 1996;59:1328-1336.

3. Benomar A, Le Guern, Durr A, et al. Autosomal-dominant cerebellar ataxia with retinal degeneration (ADCA type II) is genetically different from ADCA type I. Ann Neurol 1994;35:439-444.

4. Harding AE. The clinical features and and classification of the late onset autosomal dominant cerebellar ataxias: a study of 11 families, including descendants of "the Drew family of Walworth". Brain 1982;105:1-28.

5. David G, Dürr A, Stevanin G, et al. Molecular and clinical correlations cerebellar ataxia with progressive macular dystrophy (SCA7). Hum Molec Genet 1998;7:165-170.

6. Linhares SC, Marques W Jr, Horta W. Family princeps' history, genealogy and geographical distribution. Arq Neuropsiquiatr 2006;64:222-227.

7. Sakai T, Kawakami H. Machado-Joseph disease: a proposal of spastic paraplegic subtype. Neurology 1994;46:846-847.

8. Kaneko A, Narabayashi Y, Itokawa K, et al. A case of Machado-Joseph disease presenting with spastic paraparesis. J Neurol Neurosurg Psychiatry 1997;62:542-543.

9. Teive HAG, Iwamoto FM, Camargo CH, Lopes-Cendes I, Werneck LC. Machado-Joseph disease versus hereditary spastic paraplegia. Arq Neuropsiquiatr 2001;59:809-811.

10. Sequeiros J, Coutinho P. Epidemiology and clinical aspects of MachadoJoseph disease. In Harding AE, Deufel T (Eds). Advanceds in neurology. Inherited ataxias. Vol.61. New York: Raven Press, 1993:139-153.

11. Johansson J, Forsgren J, Sandgren O, Brice A, Holmgren G, Holmberg M. Expanded CAG repeat in Swedish spinocerebellar ataxia type 7 (SCA7) patients: effect of CAG repeat length on the clinical manifestation. Hum. Molec. Genet. 1998;7:171-176.

12. McDermot CJ, White K, Bushby K, Shaw Pj. Hereditary espastic paraparesis: a review of new development. J Neurol Neurosurg Psychiatry 2000;69:150-160.

13. Harding AE. Hereditary pure spastic paraplegia: a clinical and genetic study of 22 families. J Neurol Neurosurg Psychiatry 1981;44:871-883. 